\title{
Increased Placental Syncytial Knots
}

National Cancer Institute

\section{Source}

National Cancer Institute. Increased Placental Syncytial Knots. NCI Thesaurus. Code C118144.

Increased number of syncytiotrophoblasts with clusters of apoptotic bodies compared to what would be expected for gestational age. 